\title{
Re: Cross-Sectional and Longitudinal Associations of Sexual Function with Urinary Tract Symptoms in Men with Benign Prostatic Hyperplasia
}

\author{
Fwu C-W, Kirkali Z., McVary K. T., Burrows P. K., Eggers P. W., Kusek J. W.
}

Journal Of Urology 2015 Jan;193(1):231-238.

\section{EDITORIAL COMMENT}

In this study authors examine the cross-sectional associations between baseline characteristics and sexual function and the longitudinal associations between change in lower urinary tract symptoms and change in sexual function among men with benign prostatic hyperplasia. The cross-sectional cohort included 2.916 men who completed Brief Male Sexual Function Inventory (BMSFI) at baseline. The longitudinal cohort included 672 men who were randomized to placebo. Increased age, less education, obesity and severe lower urinary tract symptoms were found significantly associated poorer sexual drive, erectile dysfunction, ejaculatory function, sexual problem assessment and overall satisfaction. However, none of these baseline characteristics predicted change in sexual function in the longitudinal cohort. The decline in sexual dysfunction associated with worsening of lower urinary tract symptoms in men assigned to placebo was small.

Emre Bakırcıoğlu MD

\section{Re: The Effect of Sperm DNA Fragmentation on Live Birth Rate after IVF or ICSI: A Systematic Review and Meta-Analysis}

\author{
Osman A., Alsomait H., Seshadri S., El-Toukhy T., Khalaf
}

Reproductive BioMedicine online 2015 Feb;30(2):120-127.

\section{EDITORIAL COMMENT}

We need valid diagnostic tests to evaluate male infertility that add to the information more than routine semen analysis. It is necessary not only our clinical decisions for the treatment of the couple but also understand the reason that may decrease their fertility. For many years, several tests have been proposed for clinical assessment of semen quality, including computer-derived motility measures, morphological assessments (morphometry, vacuoles, Kruger's evaluation), chromatin compaction, apoptotic markers and others. Some of these measures are used in clinical practice, but few are used routinely. Many clinicians remain undecided regarding the clinical value of DNA fragmentation testing of spermatozoa. It is not clearly understand if DNA fragmentation test results change the routine practice and provide better information than the high-quality semen analysis.

Osman et al. report the results of a meta-analysis of studies that used live birth rate as an endpoint for patients undergoing IVF or IVF-ICSI. Of the three papers based on live birth rate that controlled for female fertility, an association was found between low DNA fragmentation and live birth rate following IVF, but in two other studies, no association with live birth rate following ICSI was found. The drawbacks of the studies were variable patient population, non-consecutive enrollment, lack of control for female infertility, and variable DNA fragmentation methodology and cut-off levels. It was reported that finding an association is not strong evidence that DNA fragmentation test will provide information to suggest that IVF or IVF-ICSI will result in greater success. Additional studies with large sample sizes, appropriate study populations and standardized methods are needed to confirm that DNA fragmentation testing is able to diagnose infertility and suggest which intervention is appropriate. 